\title{
On the Connection between the Dynamical System and the Ellis Compactification with Transitive Pointed System
}

\author{
Lieth A. Majed
}

Received 28/12/2016

Accepted 12/6/2017

This work is licensed under a Creative Commons Attribution 4.0 International License.

\begin{abstract}
:
In this paper, some relations between the flows and the Enveloping Semi-group were studied. It allows to associate some properties on the topological compactification to any pointed flows. These relations enable us to study a number of the properties of the principles of flows corresponding with using algebric properties. Also in this paper proofs to some theorems of these relations are given.
\end{abstract}

Keywords: Dynamical system, semi-topological semi-group, isomorphisim of $\mathrm{H}$-systems, monoidal compactification, transitive pointed systems, inclusive transitive pointed systems.

\section{Introduction:}

Historically the Enveloping semi-group which is a closure of the set of continuous functions on a compact space $\mathrm{X}$ was used to study the dynamical system as given by R.Ellis [1]. In 1991 A. Lisan characterized what is called transitive flows which is related with the right topological monidal compactification of the system [2]. In 1992 J. D. Lawson showed that many typical of lows can be recognized by restrictions on the minimal ideals of the enveloping semi-group[3]. In 2013, N. Hindman studied the intersection between the stonecech compactification with the additive and multiplication operations to present conditions on system that makes the closure of the minimal ideal of stone-cech compactification a right ideal in the case of multiplication operation [4]. Recently in 2014 L. Jones pointed out some close relation between the theory of right topological semi-group and the theory of systems on compact spaces.

The Enveloping semi-group has been established to be an essential tool in the abstract theory of topological dynamical systems. We study this with respect to enveloping semi-group. We are looking to what we called inclusive compactification which is topologically and algebraically isomorphic to the enveloping semi-group, study this by using semigroup property of the universal compactification and give some properties related to these concepts.

Department of Mathematics, College of Science, University of Diyala, Diyala, Iraq.

E-mail: liethen84@yahoo.com

\section{Preliminaries:}

Let $\mathrm{H}$ be a semi-group given with Hausdorff space. For $m, n \in H$ we define $f_{n}(m)=m n$ with product $m n$ [5]. The function $f_{n}$ is a right continuous. The semigroup $\mathrm{H}$ is called the right topology. If all $f_{n}$ are continuous then the semigroup is semi topological [6]. Given $\mathrm{H}$ be a semi-topological semi-group, an $\mathrm{H}$-system or $\mathrm{H}$ flow is a triple $(H, X, g)$, where $X$ is a compact Hausdorff space and $g: H \times X \rightarrow X$ is separately continuous[5]. Let $g(h, x)=h x$, and $g$ is defined an action if $(h r) x=h(r x)$ for all $h, r \in H$ and $x \in X$ [7].

Suppose $\mathrm{X}$ and $\mathrm{Y}$ be two $\mathrm{H}$-systems, a continuous function $T: X \rightarrow Y$ satisfying $T(h x)=h T(x)$ for all $h \in H, x \in X$ is called a homomorphism. $g: X \rightarrow Y$ is an isomorphism $\mathrm{H}$-systems if it is onto homeomorphism[8]. We define the orbit of y for $y \in X$ to be the set $H y=\{h y: h \in H\}$ and we will denote $\mho(y)$ to be the topological closure of $H y$. If there is a $y \in X$ s.t $\{y\} \cup \mho(y)=X$ we call it the system pointed transitive[4].

\section{Definition(2.1):[3]}

A monoidal right topological compactification of $\mathrm{H}$ (where $\mathrm{H}$ has an identity, otherwise one can attach an identity to $\mathrm{H}$ ) is a pair $(T, f)$ s.t

(a) $\mathrm{T}$ is Hausdorff compact right topological semigroup which contains an identity.

(b) $\mathrm{f}$ is a homomorphism continuous which takes $\mathrm{H}$ into $\mathrm{T}$. 
(c) Let e be the identity element of T, $f(H) \cup\{e\}$ is dense in $\mathrm{T}$.

(d) $\mathrm{f}$ takes the identity of $\mathrm{H}$ to the identity of $\mathrm{T}$.

(e) $f(h) \subseteq\left\{x \in T: g_{x}(y)=x y\right.$ is continuous $\}$.

By standard compactification arguments there exists an inclusive right topological compactification $(\mathrm{CH}, j)$ of $\mathrm{H}$ which is distinguished by the universal property that if $f: H \rightarrow T$ is a right topological compactification of $\mathrm{H}$, then there exist a unique continuous homommorphism a $F: C H \rightarrow T$ s.t $f=F \circ j$.

Theorem(2.2): Let $(X, q)$ be a pointed transitive Hsystem and $(Y, p)$ be a pointed $\mathrm{H}$-system. Then there is at most one homomorphism from $(X, q)$ to $(Y, p)$.

Proof: See Proposition 1-1 in[4].

$\operatorname{Remark}(2.3)$ : Let $(X, q)$ and $(Y, p)$ be two pointed transitive systems, if $h:(X, q) \rightarrow(Y, p)$ and $r:(Y, p) \rightarrow(X, q)$ are homomorphism, then $\mathrm{h}$ and $\mathrm{r}$ are inverse homeomorphism and therefore $\mathrm{H}$ system is an isomorphism[9].

$\operatorname{Remark}(2.4)$ : Let (H, X, D) be an H-system. For each $h \in H$, let $D^{*}$ denote the continuouse mapping $x \mapsto h x$, we fram the Ellis semigroup by capturing the closure of $H(D)=\left\{D^{*}: h \in H\right\}$ in the Cartesian product $X \times X$ given with the product topology.

\section{Main results:}

In this part, we present the new definitions and theorems related with the study and the relations between the dynamical system the enveloping semigroup.

Definition(3.1):[2]

Consider $\left(T_{1}, d_{1}\right)$ and $\left(T_{2}, d_{2}\right)$ be two monidal compactifications of $\mathrm{H}$. We define the $\mathrm{C}$ homomorphism $K: T_{1} \rightarrow T_{2}$ which is a continuous homomorphism identity preserving such that $K \circ d_{1}=d_{2}$.

Lemma(3.2): Let $\left(T_{1}, d_{1}\right)$ and $\left(T_{2}, d_{2}\right)$ be monidal compactification then $\mathrm{K}$ is a homomorphism of pointed $\mathrm{H}$-system iff $K:\left(T_{1}, d_{1}\right) \rightarrow\left(T_{2}, d_{2}\right)$ is a Chomomorphism.

Proof: If $\mathrm{K}$ is a homomorphism pointed of $\mathrm{H}$ systems, then for $h \in H, n \in T_{1}$ we get $K\left(d_{1}(h) n\right)=K(h n)=h K(n)=$ $d_{2}(h) K(n) \ldots .(1)$.

Take $\mathrm{n}=1$, we have $K \circ d_{1}=d_{2}$ and thus $K \circ$ $d_{1}(h)=d_{2}(h)$ for all $h \in H$. Let $n, m \in T_{1}$. Since $m \in T_{1}$, then there is a net $\left\{d_{1}\left(h_{\alpha}\right) n\right.$ converge to $\mathrm{nm}$. On the other hand, $d_{1}\left(h_{\alpha}\right) n=$ $d_{2}\left(h_{\alpha}\right) K(n) b y(1)$

$=K\left(d_{2}\left(h_{\alpha}\right)\right) K(n)$.

But by continuity of $\mathrm{K}$ we get $K\left(d_{1}\left(h_{\alpha}\right)\right)$ convergence to $\mathrm{K}(\mathrm{m})$ and so $K\left(d_{1}\left(h_{\alpha}\right)\right) K(n)$. Thus $K(m n)=K(m) K(n)$.
Conversely, by definition (2), $K \circ d_{1}=d_{2}$. Therefore

$K(n x)=K\left(d_{1}(h) n\right)=K\left(d_{1}(h)\right) k(x)=$ $d_{2}(h) K(x)$. Hence $\mathrm{K}$ is a homomorphism of pointed $\mathrm{H}$-system.

Remark(3.3): $\mathrm{T}$ can be considered as an $\mathrm{H}$-system by defining $T(h, t)=j(h) t$ where $\mathrm{H}$ is topological semi-group and $(T, j)$ be a monoidal compactification.

Lemma(3.4): Consider $(H, X, D)$ as an H-system then

(a) $f \mapsto f(q): V(X) \rightarrow X \quad$ where $q \in X$, is a homomorphisim of H-systems, and a homomorphisim of pointed $\mathrm{H}$-systems, from $\left(V(X), I_{x}\right)$, where $I_{x}$ is the identity function.

(b) If $(T, j)$ is a monoidal compactification of $\mathrm{H}$ defined on part (a) from $\left(V(T), 1_{T}\right)$ to the flow $(T, 1)$ is an isomorphic, where 1 is the identity of $T$.

Proof: For part (a). Let $R: V(X) \rightarrow X$ which is defined by $R(f)=f(q)$ for $q \in X$, then $R(h f)=$ $(h f)(q)=s f(q)=h R(f)$ for all $h \in H$ and $f \in V(x)$. Also $R\left(\operatorname{Id}_{x}\right)=I_{x}(q)=q$. Thus $\mathrm{R}$ is homomorphisim pointed $\mathrm{H}$-system. For part (b) is left for the reader.

Remark(3.5): Let $(H, X)$ and $(H, Y)$ be two systems, if $K$ is surjective homomorphisim from $X$ to $Y$ map then $R: V(X) \rightarrow V(Y)$ is unique homomorphisim which is defined by $R(a)=$ $K(a(x)$, where $x \in X$ satisfies $K(x)=y$.

Theorem (3.6): Let $R: V(X) \rightarrow Y$ be the flow homomorphisim $h \mapsto h q$ Then $\mathrm{R}$ is an isomorphism of flows iff $(X, q)$ is isomorphic to the flow coming from some monidal compaction $(T, j)$.

Proof: Clearly $(X, q)$ must be a pointed H-system. Let $\mathrm{R}$ be an isomorphic of flows. Thus $(X, q)$ is an isomorphic to the Ellis compactification. On the other hand, assume that $(X, q)$ is isomorphic for some monidal compactification $(T, j)$. We can define an isomorphism $Z: V(X) \rightarrow V(Y)$ as in remark (4). Therefore $(X, q)$ is isomorphic to its Ellis compactification $\left(V(X), I_{x}\right)$.

Definition(3.7):

A pointed transitive $\mathrm{H}$-system $(X, p)$ is said to be inclusive if there is a homomorphisim from $(X, p)$ to any other pointed $\mathrm{H}$-system.

Definition(3.8): [9]

$\operatorname{Let}(H,+)$ be a semigroup, for any $A \subseteq H$ and $x \in H$ we define $-x+A=\{h \in H: x+h \in A\}$. Given any two ultrafilters $p, q \in C H$ we define their sum by $p+q=\{A \subseteq H /\{x \in H /-x+A \in q\} \in$ $p\}$.

In the next Theorem, we will see how the inclusive right topological compactification of $\mathrm{H}$ led to the inclusive pointed transitive $\mathrm{H}$-flow $(\mathrm{CH}, \overline{\mathrm{e}})$. 
Theorem (3.9): Let $(\mathrm{CH}, j)$ be the inclusive right topological compactification of $\mathrm{H}$. Let we define $K: H \times C H \rightarrow C H$ by $k(n, q)=j(n) q$ where the right hand side is added in $\mathrm{CH}$. Then $(\mathrm{H}, \mathrm{CH}, \mathrm{K})$ is an $\mathrm{H}$-system, and the pointed H-system $(\mathrm{CH}, \overline{\mathrm{e}})$ where $\bar{e}$ is the base point.

Proof: Note that by definition of filter addition above we have $K(n, q)=j(n)+q=f_{j(n)}(q)$ where $f_{j(n)}$ right continuous. Thus $\mathrm{K}$ is separately continuous. Since $\mathrm{j}$ is a homomorphism, $K\left(n_{1}+\right.$ $\left.n_{2}, q\right)=j\left(n_{1}+n_{2}\right) q=j\left(n_{1}\right)+j\left(n_{2}\right) x=$ $j\left(n_{1}\right) j\left(n_{2}\right) x=j\left(n_{1}\right) n_{2} q=K\left(n_{1}, n_{2} q\right)$. Therefore, $\mathrm{K}$ is defined as an action of $\mathrm{H}$ on $\mathrm{CH}$. Since $j(\Theta)=\bar{\Theta}$ then $K(\Theta, x)=K(\Subset) x=\bar{\Theta} x=\Subset x=x$ acts as an identity on $\mathrm{CH}$. Also $K(H \times\{\overline{\mathbb{e}}\}) \cup$ $\{\overline{\mathbb{e}}\}=j(H) \overline{\mathbb{e}} \cup\{\overline{\mathrm{e}}\}=j(H) \cup\{\overline{\mathrm{e}}\}$ is dense in $\mathrm{CH}$, implies the action is a point transitive. To show $(C H, \bar{\Theta})$ is inclusive. Let $(Y, p)$ be a pointed $\mathrm{H}$ system. Thus, the extended action $\bar{K}: C H \times Y \rightarrow Y$ is right continuous action of $\mathrm{CH}$ on Y. Define $\tau: C H \rightarrow Y$ by $\tau(t)=t p$, where the right hand side is the extended action. Clearly $g$ preserves distinguished points. Finally, for $\kappa \in H$ and since $\mathrm{j}$ is a homomorphisim then $\tau(\kappa x)=\tau(j(\kappa) x)$ $=\tau(j(\kappa) j(r))$ where $j(r)=x$

$=\tau(j(\kappa+r))$

$=j(\kappa+r) q$

$$
\begin{aligned}
& =j(\kappa) j(r) q \\
& =\kappa \tau(r) \\
& =\kappa \tau(j(r)) \\
& =\kappa \tau(x) .
\end{aligned}
$$

Thus $\mathrm{g}$ is a homomorphism of $\mathrm{H}$-system and the proof is complete.

\section{References:}

[1] Devaney, R. L. 1989. Chaotic dynamical Systems, Second Edition, addition-Wesley, New York.

[2] Lawson, J. D. and Lisan, A. 1991. Transitive Flows. A semi-group Approach, Mathematica 38: 348-361.

[3] Lawson, J. D. Flows and Compactifications, J. 1992. London math. Soc.(2) 46: 349-363.

[4] Auslander, J. 1988. Minimal Flows and Their Extensions, North-Holland, Amsterdam.

[5] N.Hindman and D. Strauss. Algebra in the StoneCech Compactification Theory and Applications, Second Edition, 2010.

[6] Munkres, J. R. 2000. Topology Second Edition, Prentice hall, Inc., NJ.

[7] Hindman , N. and Bergelson, V. 2012. Quotient sets and density recurrent sets, Trans. Amer. Math. Soc. 364: 4495-4531.

[8] Hindman , N. and Jones, L. 2014. Idempotents in $\beta S$ that are only products trivially, New York J. Math. 20:57-80.

[9] Hindman, N. and Phulara, D. 2013. Some new additive and multiplicative Ramsey numbers $\mathrm{J}$. Combinatorics 4: 81-93.

\section{العلاقة بين النظام الديناميكي مع تراص ايليس والنظام الديناميكي المتعدي الحاوي على نقطة اساسية \\ ليث عبد الطيف مجيد}

قسم الرياضيات، كلية العلوم، جامعة ديالى، ديالى، العراق.

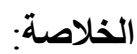

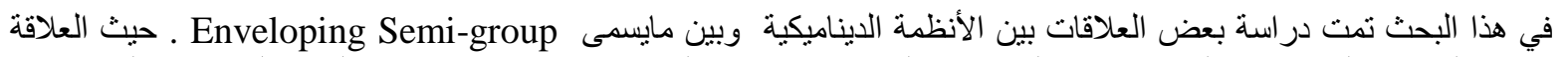

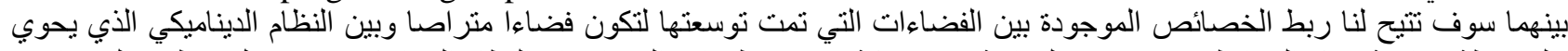

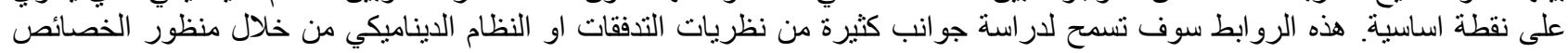
الجبرية.

الكلمات المفتاحية: النظام الديناميكي، شبه التبولوجي شبه الزمرة، تماتل الأنظمة H، نظام النقطة التتعدي، انظمة ذات قاعدة شاملة. 